\title{
Is Inhibition of Return Modulated by Involuntary Orienting of Spatial Attention: An ERP Study
}

\author{
Fada Pan *, Xiaogang Wu $\mathrm{Wu}^{+}$and Li Zhang \\ The Department of Applied Psychology, School of Education Science, Nantong University, Nantong, China
}

\section{OPEN ACCESS}

Edited by:

Ramesh Kumar Mishra, University of Hyderabad, India

Reviewed by:

John J. McDonald,

Simon Fraser University, Canada

Juan Lupiáñez,

University of Granada, Spain

*Correspondence:

Fada Pan

psyc_lee2015@126.com.

tThese authors have contributed equally to this work.

Specialty section: This article was submitted to Cognition,

a section of the journal

Frontiers in Psychology

Received: 12 September 2016 Accepted: 17 January 2017 Published: 31 January 2017

Citation:

Pan F, Wu X and Zhang L (2017) Is Inhibition of Return Modulated by Involuntary Orienting of Spatial

Attention: An ERP Study.

Front. Psychol. 8:113.

doi: 10.3389/fpsyg.2017.00113
Inhibition of return (IOR) is a mechanism that indicates individuals' faster responses or higher accuracy to targets appearing in the novel location relative to the cued location. According to the "reorienting hypothesis," disengagement from the cued location is necessary for the generation of IOR. However, more and more studies have questioned this theory because of dissociation between voluntary or involuntary spatial orienting and the IOR effect. To further explore the "reorienting hypothesis" of IOR, the present experiment employed an atypical cue-target paradigm which combined a spatially nonpredictive peripheral cue that was presumed to trigger IOR with a spatially non-predictive central cue that was used to reflexively trigger a shift of attention. The results showed that a significant IOR effect did not interact with automatic spatial orienting as measured in mean RTs and accuracy as well as the Nd component. These findings suggested that the IOR effect triggered by peripheral cue was independent of automatic orienting generated by a central cue. Therefore, the present study provided evidence from location task and neural aspects, which again challenged the "reorienting hypothesis" of IOR.

Keywords: inhibition of return, reorienting hypothesis, peripheral cue, central cue, event-related potentials

\section{INTRODUCTION}

Attention orienting can be induced voluntarily or involuntarily by a variety of stimuli. Traditional literature has described two different mechanisms: endogenous orienting and exogenous orienting, which represented inner goals and external demands respectively (Jonides, 1981; Posner and Cohen, 1984; Klein, 2000; Chica et al., 2011). The common operation is that researchers present a predictive central cue (e.g., an eye-gaze or arrow that points to the left or right) at fixation to study endogenous orienting, and present a non-predictive peripheral cue in the left or right to study exogenous orienting (Ruz and Castillo, 2002). Orienting of attention in both types of cues involves three processes of engagement, disengagement and re-orienting, and the cue-target paradigm has been widely used to investigate these processes (Posner, 1980).

Typically, a non-informative peripheral cue can produce facilitatory effect or inhibitory effect based on different stimulus onset asynchronies (SOA). If the cue-target SOA is less than $300 \mathrm{~ms}$, a facilitatory effect can be observed, showing faster reaction times (RTs) at the cued location (cue and target appear on the same side) than those at the uncued location (cue and target appear on different sides). On the contrary, if the cue-target SOA is longer than $300 \mathrm{~ms}$, early facilitatory effect will be replaced by an inhibitory effect. That is the well-known inhibition of return (IOR) (Posner et al., 1985), which refers to the faster response at the uncued location than that at the 
cued location (Posner and Cohen, 1984). However, when the central cue is presented at fixation, a long-lasting facilitatory effect can be observed even at a longer SOA (e.g., $1000 \mathrm{~ms}$ ): individuals generally respond faster to targets appearing at a location indicated by the central cue than those not indicated by a central cue (Funes et al., 2007). Psychologists explain that IOR is a mechanism reflecting adaptability and flexibility of the human cognitive process by encouraging attention shift to a novel location and inhibiting attention from re-orienting back to an inspected location (MacInnes and Klein, 2003; Ivanoff and Taylor, 2006). According to this "re-orienting hypothesis," attentional disengagement is essential for generating the IOR effect: when the attention is attracted by a peripheral cue, the cued location first produces a facilitatory effect; then, after a few $100 \mathrm{~ms}$, the attention is disengaged from the cued location and an inhibitory effect starts to prevent the attention from returning to that cued location.

Even though the explanation of the "re-orienting hypothesis" of IOR was widely accepted by many researchers in the attention field, many studies have focused on the voluntary attention and they found a robust IOR effect when attentional disengagement did not take place. Researchers used predictive peripheral cues to guarantee that indicated and non-indicated trials can be either cued or uncued in both detection and discrimination tasks (Chica et al., 2006). The results showed that a typical IOR effect was consistently found at the indicated location. Berger et al. (2005) used a paradigm with a predictive central cue and a non-predictive peripheral cue to investigate the relation between IOR and voluntary orienting (a predictive central cue) of visual attention. In the experiment, the validity of an endogenously predictive central cue was $80 \%$ (i.e., the target appeared at the indicated location with $80 \%$ probability). The IOR effect was observed not only at the indicated location but also at the non-indicated location. Moreover, there is a growing body of work showing that the disengaging of attention seems to be unnecessary or insufficient (Danziger and Kingstone, 1999; Chica and Lupiáñez, 2004, 2009; Lupiáñez, 2010, chapter 2).

Recently, Martín-Arévalo et al. (2013) combined both nonpredictive peripheral cue and non-predictive central cue to demonstrate the separation from the IOR effect and involuntary orienting of spatial attention. In their experiments, the peripheral cue was manipulated by presenting a salient cue in one of four positions (up, down, left, and right), and involuntary orienting was operated by the central cue pointing to one of the four potential target locations. The results were clear: no matter how the central cue was operated, the interactions between peripheral cue and central cue didn't reach a significant level (Martín-Arévalo et al., 2013). The set of implied cues (e.g., gazing face, gazing eye or arrow) had a significant facilitatory effect indicating reflexive orienting to the target location (Friesen and Kingstone, 2003). Although centrally fixated directional stimuli like gaze cues and arrow cues have been demonstrated to the generation of reflexive orienting with long or short SOA (Friesen and Kingstone, 1998; Tipples, 2002), Green and Woldorff (2012) raised a spatial-conflict explanation when they used predictive arrow cues to find large RT cueing effects at very short cue target intervals (Green and Woldorff, 2012). The conflict between the cue and the target among invalid trials might be induced by the persistent cue. Then, Green et al. (2013) examined the time course of uninformative-arrowcue effect and found that no significant cueing effect was observed for both short-duration conditions and significant cueing effect was observed at the two shortest SOA for the long-duration cues. The cueing effect was only found in the temporal overlap of the cue and target (long duration). They suggested that their results supported a spatial-incongruency explanation rather than automatic attention-orienting accounts. Gayzur et al. (2014) also examined the time course of uninformative-gaze-cues and found that short duration cues led to facilitation at short SOA but not long SOA and long duration cues induced the cueing effect at short and long SOA (interpreted as automatic orienting). Martín-Arévalo et al. (2013) manipulated the long duration arrow cue at the long SOA and they found RT was faster when target appeared at the indicated position, reflecting automatically attentional allocation of indicated location. It seemed that reflexive orienting was related with long duration in which central cues overlapped temporally with the target at the long SOA, when spatialconflict was related with long duration at the short SOA. Regardless of different interpretations of the central cueing effect, IOR can occur when the attention was disengaged voluntarily or involuntarily from the attended location. Although there were some evidences which argued against the "re-orienting hypothesis" of IOR, further experiments using different tasks or paradigms are required to improve the validity of this disagreement.

To further investigate the modulation of the validity effect induced by the central arrow cue over the IOR effect, in the atypical paradigm of Martín-Arévalo et al. (2013), the present experiment used the same procedure. The purpose of the present study was to replicate previous results (Berger et al., 2005; Chica et al., 2006; Martín-Arévalo et al., 2013) and further explore the neural correlates of dissociation between the IOR effect and automatically attentional orienting. Firstly, we employed an atypical cue-target paradigm similar to the one used by MartínArévalo et al. (2013), in which the non-predictive peripheral cue was presented before the non-informative central cue. However, in the current experiment, we modified the procedure into a more common IOR paradigm (i.e., only the left and right locations). Additionally, we chose only the arrow cue to orient attention. Motoric and visual were two forms of IOR relating to activation and suppression of the oculomotor system, respectively (Taylor and Klein, 2000). Suppression of the oculomotor system often uses a localization task and a detection task, during which participants are required to keep their eyes on a fixation point. In the present experiment, a localization task was used instead of a detection task (Martín-Arévalo et al., 2013).

Our second aim was to use event-related potentials (ERPs) recording to understand the combination of automatic attentional orienting and the IOR effect. The ERP technique can provide more valuable information of the potential process in addition to the results of behavioral experiments. Moreover, 
as there is insufficient evidence about electrophysiological correlates of IOR and involuntary spatial orienting, the method can also investigate the consistency of behavioral results and ERP results. The current experiment sought to investigate the early stage of IOR with sensory/perception component in the visual spatial attention field. Electrophysiological components like $\mathrm{P} 1, \mathrm{~N} 1, \mathrm{Nd}$, and $\mathrm{N} 2 \mathrm{pc}$ were used to investigate the modulations of peripheral cueing associated with behavioral facilitation or inhibition effects for different research purposes. An enhancement of the P1 component (larger amplitude for cued location trials than uncued ones) was usually associated with the facilitatory effect (Doallo et al., 2004), while a reduction of the P1 component was associated with the IOR effect (McDonald et al., 1999; Prime and Jolicoeur, 2009; Satel et al., 2013). Additionally, the behavioral IOR effect has been observed in the absence of the P1 effect (Wang et al., 2012). Therefore, researchers have concluded that P1 may not be a direct marker of IOR (Prime and Ward, 2006; Jones and Forster, 2012, 2014; Satel et al., 2014). The facilitatory effect associated with N1 may be enhanced for cued trials rather than uncued ones (Eimer, 1994; Martín-Arévalo et al., 2014), and the IOR effect had a reversal with enhanced amplitude of N1 for uncued rather than cued trials (Prime and Ward, 2004; Prime and Jolicoeur, 2009; Satel et al., 2014). However, there was still an inconsistent association between $\mathrm{N} 1$ and behavioral cueing effects (Hopfinger and Mangun, 1998; Prime and Ward, 2006; Chica and Lupiáñez, 2009). The Nd component can be observed in most experiments, but there was no agreed upon explanation of the underlying mechanism. Researchers found a potential association that may reflect the IOR effect of $\mathrm{Nd}$ at the occipital electrodes (Satel et al., 2013, 2014), which refers to a reduction of the $\mathrm{Nd}$ component at the cued location. The first N2pc study of IOR was investigated in the covert deployment of attention (McDonald et al., 2009). They found reduced amplitude at the cued location relative to the uncued location and explained that the inhibitory process underlying IOR reduced the probability of attention returning to cued location. Recently, researchers found a reduction of the N2pc component associated with spatial attention of the IOR effect on the cued trials (Martín-Arévalo et al., 2014). Having said that, further research should be conducted due to the lack of experimental data of N2pc. Since behavioral predictions and electrophysiological modulations related to facilitation and IOR effects depend on many variables, there was no single electrophysiological marker for facilitation and IOR (Martín-Arévalo et al., 2016).

Considering that IOR is independent of either voluntary or involuntary spatial orienting (Chica and Lupiáñez, 2009; Martín-Arévalo et al., 2013), we hypothesize that IOR will not be mediated by non-predictive central cues. Another crucial hypothesis is that exogenous (peripheral cue) and endogenous (central cue) orienting mechanisms may contribute independently to performance and each mechanism has its own representative and independent effect (Berger et al., 2005; Chica et al., 2013). In line with behavioral outcomes, there will also be a disassociation between IOR and spatial orienting at the neural level.

\section{MATERIALS AND METHODS}

"This study was approved by the Human Ethics Committee of Nantong University. A written informed consent was obtained from all the participants before their participation."

\section{Participants}

Twenty three paid university participants (13 female, 10 male; aged 19-23 years, mean age 21) volunteered to participate in the study. All participants had normal or corrected-to-normal vision, were right-handed, were without mental illness and color blindness, and hadn't participated in a similar experiment before.

\section{Stimuli and Apparatus}

All stimuli were drawn in white and presented on a black background. Each trial started with two rectangular boxes and a fixation. Each box was $2.34^{\circ}$ (horizontal) $\times 3.36^{\circ}$ (vertical) and fixation was $0.5^{\circ}$ (horizontal) $\times 0.5^{\circ}$ (vertical). The peripheral cue was a solid circle appearing centrally in one of two rectangular boxes. The central cue was represented by an arrow with an arrowhead and arrow-tail (pointing to the right or left) or a straight line (catch trials). The target was a hollow circle, the same size as the peripheral cue, and appearing in the right or left box. Participants sat in a dimly lit and electromagnetically shielded room, with their heads located $70 \mathrm{~cm}$ away from the computer screen. The experiment was presented with E-prime software, which controlled the presentation of the stimuli and the acquisition of data on a PC.

\section{Design and Procedure}

The present experiment consisted of a 2 (peripheral cueing: cued vs. uncued) $\times 2$ (central cueing: indicated vs. non-indicated) within-subject design. When the cue and target appeared at the same location, it was named cued trials. When the cue and target appeared at different locations, it was named uncued trials. An indicated or non-indicated trial referred to a target appearing at the location that was, or was not, indicated by the central cue. In the course of the experiment, participants were asked to locate the target, and their RTs, correct rate, and EEG data were recorded simultaneously.

Before entering the laboratory, participants were explained the basic principles of ERP recording in order to eliminate their tension. They were then required to give written informed consent. The experimental procedure is depicted in Figure $\mathbf{1}$. Each trial began with the presentation of central fixation and two rectangular boxes with a variable duration between 750 and $850 \mathrm{~ms}$. Participants needed to maintain their fixation on a cross during the experiment. In one of two possible boxes, a non-predictive cue (solid circle) was presented randomly with equal probability for $200 \mathrm{~ms}$. After a $300 \mathrm{~ms}$ delay, the cross fixation was replaced by the central cue (arrow or straight line) for $300 \mathrm{~ms}$. The central cue appeared with equal probability toward the left or right and was non-predictive for the target location. Subsequently, after another $300 \mathrm{~ms}$ delay, the target (hollow circle) appeared in the left or right box with equal probability until participants made a response or $1000 \mathrm{~ms}$ had elapsed. Participants were asked to judge the target location as 


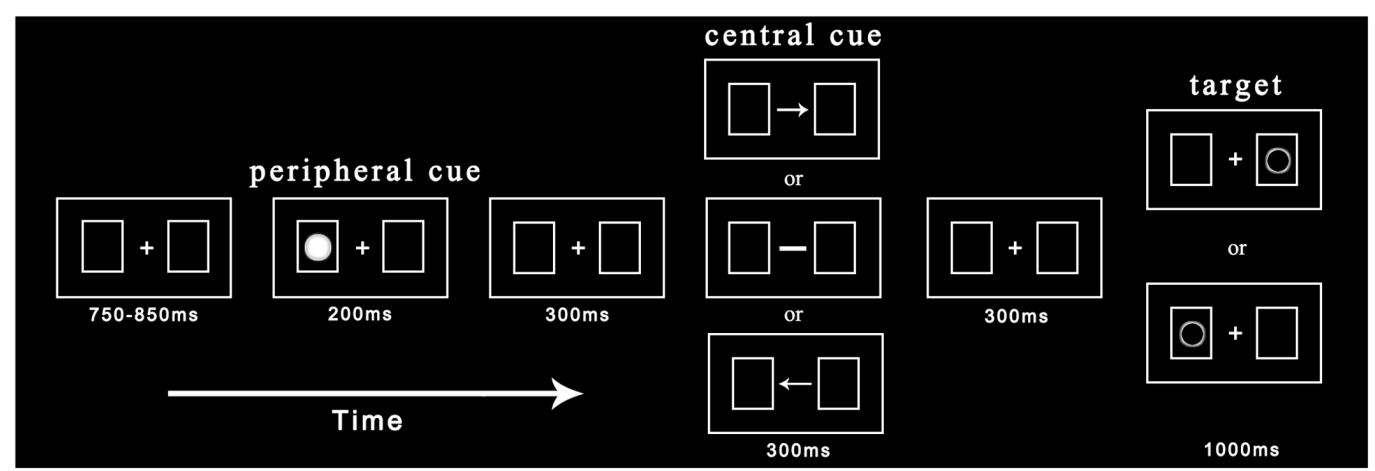

FIGURE 1 | Procedure of events in a sample trial. The peripheral cue and target appear randomly at the left or right location with equal probability while the central cue points to the left or right. Under indicated and non-indicated conditions, cued and uncued locations have the same number of trials.

soon as they found the target: if the target appeared in the left box, participants were instructed to press the " $Z$ " key; if the target appeared in the right box, participants were instructed to press the "M" key. Each trial ended with a black blank screen for $2000 \mathrm{~ms}$.

Prior to formal experimental blocks, each participant needed to complete practice trials. There were a total of 24 trials per practice block for each participant. The formal experiment would not start until the participants understood the experimental requirements. This would enable them to have a correct rate of at least $90 \%$. The formal experiment contained four blocks of 416 trials. Each block had 104 trials, of which 8 were catch trials (under this condition, no target was presented and no response was needed). The remaining 96 trials consisted of 24 repetitions of the factorial combination of peripheral cueing and central cueing. Neither practice trials nor catch trials were analyzed. During the experiment, participants were told to try their best to reduce their actions of swallowing, frowning, blinking, and to keep their bodies from moving. Rest periods were provided at every block.

\section{ERP Recording and Analysis}

Electroencephalogram (EEG) signals were recorded using a $64 \mathrm{Ag}-\mathrm{AgCl}$ electrodes elastic cap placed according to the international 10-20 system and the Neuroscan ERP workstation (scan4.5), with the reference electrode on the left mastoid (M1) and a ground electrode (GND) on the medial frontal aspect. A vertical electrooculogram (EOG) was recorded from above and below the participant's left eye, and a Horizontal EOG was recorded from the outer canthi of both eyes. All electrodes impedance was kept below $5 \mathrm{~K} \Omega$. The sampling rate and band pass were $1000 \mathrm{~Hz} / \mathrm{channel}$ and $0.05 \sim 100 \mathrm{~Hz}$. Every participant washed his or her hair in the laboratory first, and recording started until the impedance was stable (below $5 \mathrm{~K} \Omega$ ) with the conductive paste on the scalp.

Three participants were excluded because of excessive EEG artifacts (accepted less than 65\% of the trials). The EEG data recorded in the experiment was used by scan software for offline analysis. We fused EEG data with behavioral data, and the average of M1 and M2 was converted to a new reference. Ocular artifacts (mean EOG voltage exceeding $\pm 100 \mu \mathrm{V}$ ) related to blinks and vertical eye movements were removed from the EEG, and trials of incorrect or no responses were also removed at EEG epoch $(-100 \mathrm{~ms}-500 \mathrm{~ms}$, appearance of target used as zero point). After baseline correction, trials with excessive artifacts $( \pm 80 \mu \mathrm{V}$ standard) were rejected for further analysis (acceptance rate of each participant was more than $85 \%$ of trials). Finally, mean waveforms under all conditions performed a band-pass filter containing a high-pass filter of $0.1 \mathrm{~Hz}$ and a low-pass filter of $30 \mathrm{~Hz}$ (24 dB/octave) (Luck, 2014; Satel et al., 2013, 2014). According to the overall average map and previous research, the ERP components were divided by the time windows in which they occurred: P1, 80 140 ms; N1, 140 200 ms; and $\mathrm{Nd}, 200 \sim 300 \mathrm{~ms}$. We selected electrodes in the occipital areas for statistical analyses of P1, N1 (Po5, Po6, Po7, Po8, P5, P6, O1, O2) and Nd (Po7, Po8, Pz) (Prime and Ward, 2006; Prime and Jolicoeur, 2009; Satel et al., 2014). SPSS 16.0 for Windows was used for repeated measures analysis of variance of behavioral data and ERP data.

\section{RESULTS}

\section{Behavioral Results}

According to the $2 \times 2$ experimental design, we conducted statistical analyses of the mean response times and correct rate for each condition. Trials with no response, incorrect response and extreme response (RTs below $200 \mathrm{~ms}$ and above $900 \mathrm{~ms}$ ) were not included for RTs analysis (4.58\% of trials).

On correct rate, a 2 (peripheral cueing: cued vs. uncued) $\times 2$ (central cueing: indicated vs. non-indicated) repeated measures analysis of variance (ANOVA) revealed a significant main effect of peripheral cueing, $F(1,22)=11.41, p<0.01, \eta^{2}=0.34$, suggesting that IOR was observed with a higher correct rate at the uncued location $(99.5 \% \pm 0.13)$ than the cued location $(99.1 \% \pm 0.16)$. Neither main effects of central cueing nor interaction between peripheral cueing and central cueing (all $p>0.05)$ were significant. 
On mean correct RTs (see Figure 2), a similar ANOVA revealed a strongly significant main effect of peripheral cueing, $F(1,22)=46.69, p<0.0001, \eta^{2}=0.68$, indicating slower RTs at the cued location $(364 \pm 11)$ than the uncued location $(341 \pm 11)$. The central cueing effect was significant, $F(1,22)=5.95$, $p<0.05, \eta^{2}=0.21$, reflecting faster RTs at the indicated location $(349 \pm 10)$ than the non-indicated location (357 \pm 11$)$. Crucially, interaction involving peripheral cueing and central cueing was not significant, $F(1,22)=0.001, p=0.98, \eta^{2}=0$. The IOR effect sizes (cued RTs-uncued RTs) of the indicated cue $(23 \mathrm{~ms})$ and the non-indicated cue $(23 \mathrm{~ms})$ were not significant as well, $F(1,22)=0.001, p>0.05$. Thus, the peripheral cueing effect was independent of the central cueing effect.

\section{ERP Results}

There was a three factors repeated measures ANOVA for mean amplitudes of the P1 and N1 components (see Table 1): peripheral cueing (cued vs. uncued), central cueing (indicated vs. non-indicated), and laterality (ipsilateral vs. contralateral).

P1: The peripheral cueing effect revealed no main effect of peripheral cueing, $F(1,19)=1.69, p=0.21, \eta^{2}=0.08$, or central

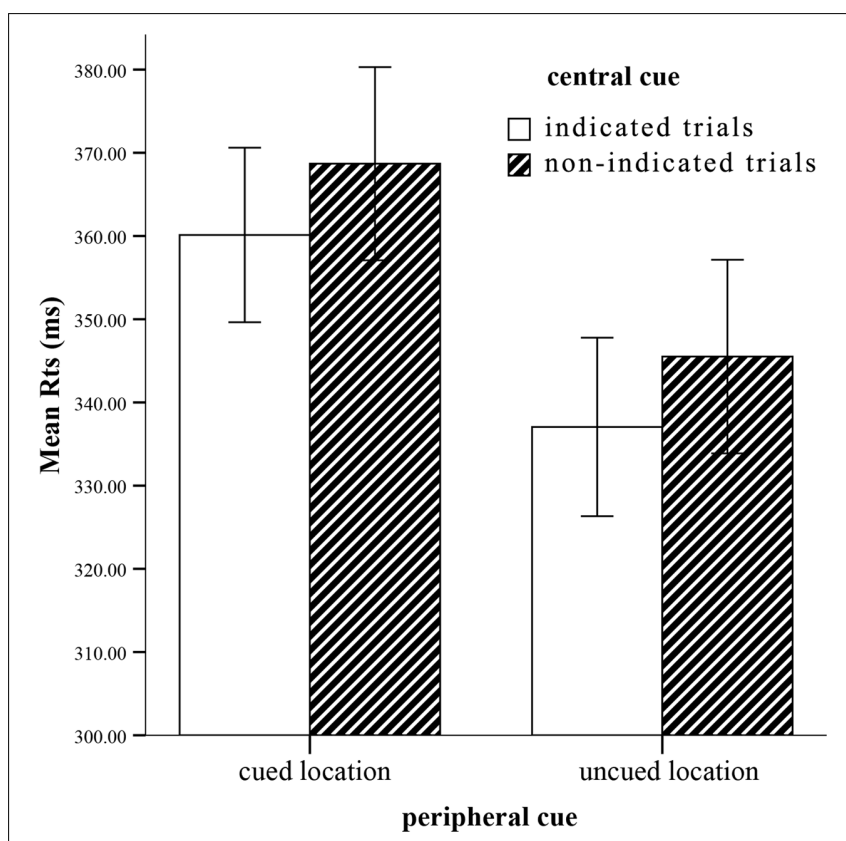

FIGURE 2 | Mean RTs for each condition. Error bars show the SE of each condition. cueing, $F(1,19)=0.01, p=0.91, \eta^{2}=0.001$, or laterality, $F(1,19)=1.68, p=0.21, \eta^{2}=0.08$, and the interactions of peripheral cueing $\times$ central cueing, $F(1,19)=0, p=0.98$, $\eta^{2}=0$, and of central cueing $\times$ laterality, $F(1,19)=1.65$, $p=0.21, \eta^{2}=0.08$, were not significant. The interaction between peripheral cueing $\times$ laterality was marginally significant, $F(1,19)=3.84, p=0.065, \eta^{2}=0.17$. The three-way interaction peripheral cueing $\times$ central cueing $\times$ laterality was also not significant, $F(1,19)=0.03, p=0.88, \eta^{2}=0.001$.

N1: The analysis showed a significant main effect of peripheral cueing, $F(1,19)=4.84, p<0.05, \eta^{2}=0.2$, with the amplitude of the uncued location $(-0.86 \mu \mathrm{V})$ being smaller than the cued location $(-1.14 \mu \mathrm{V})$. The main effect of central cueing was significant, $F(1,19)=4.72, p<0.05, \eta^{2}=0.2$, with the amplitude of the non-indicated location $(-0.79 \mu \mathrm{V})$ being smaller than the indicated location $(-1.2 \mu \mathrm{V})$. The main effect of laterality was not significant, $F(1,19)=0.85, p=0.37, \eta^{2}=0.04$. Importantly, both the interaction between peripheral cueing and central cueing, $F(1,19)=0, p=0.99, \eta^{2}=0$, and the interaction between peripheral cueing and laterality, $F(1,19)=0.1, p=0.76$, $\eta^{2}=0.01$, were not significant. The interactions of peripheral cueing $\times$ laterality, $F(1,19)=0.09, p=0.77, \eta^{2}=0.01$, and of peripheral cueing $\times$ central cueing $\times$ laterality, $F(1,19)=0.83$, $p=0.38, \eta^{2}=0.04$, were also not significant.

Mean amplitudes of the $\mathrm{Nd}$ components was assessed in a three-way, repeated-measures ANOVA with factors for peripheral cueing (cued, uncued), central cueing (indicated, nonindicated), and electrodes (Po7, Po8, Poz). The peripheral cueing effect was significant, $F(1,19)=26.47, p<0.001, \eta^{2}=0.58$, with the amplitude of the uncued location $(3.94 \mu \mathrm{V})$ being larger than the cued location $(2.85 \mu \mathrm{V})$. The central cueing effect was not significant, $F(1,19)=0.05, p=0.83, \eta^{2}=0.002$. Most importantly, the interaction between peripheral cueing and central cueing was not significant, $F(1,19)=0.17, p=0.69$, $\eta^{2}=0.009$. The interaction of peripheral cueing $\times$ electrodes, $F(2,38)=6.79, p<0.001, \eta^{2}=0.26$, was significant, showing larger cueing effects at the Poz electrodes. The interactions of central cueing $\times$ electrodes, $F(2,38)=2$, $p=0.31, \eta^{2}=0.06$, and of peripheral cueing $\times$ central cueing $\times$ electrodes, $F(2,38)=0.14, p=0.87, \eta^{2}=0.01$, were not significant.

According to the difference waves of Figure 3, mean amplitudes of an early $\mathrm{Nd}$ (during 100-200 ms latency window) were assessed in a three-way, repeated-measures ANOVA (peripheral cueing $\times$ central cueing $\times$ laterality). The results showed that, the peripheral cueing effect was significant, $F(1,19)=4.54, p<0.05, \eta^{2}=0.19$, and the ERPs were

TABLE 1 | Mean ERP component $(M \pm S E \mu V)$ and peaks (ms) for each condition.

\begin{tabular}{|c|c|c|c|c|c|c|}
\hline Cueing & \multicolumn{2}{|c|}{ P1 } & \multicolumn{2}{|c|}{ N1 } & \multicolumn{2}{|c|}{$\mathrm{Nd}$} \\
\hline Uncued & $\begin{array}{c}0.21 \pm 0.36 \\
118\end{array}$ & $\begin{array}{c}0.21 \pm 0.31 \\
115\end{array}$ & $\begin{array}{c}-1.1 \pm 0.43 \\
172\end{array}$ & $\begin{array}{c}-0.46 \pm 0.33 \\
172\end{array}$ & $3.97 \pm 0.56$ & $3.92 \pm 0.53$ \\
\hline
\end{tabular}




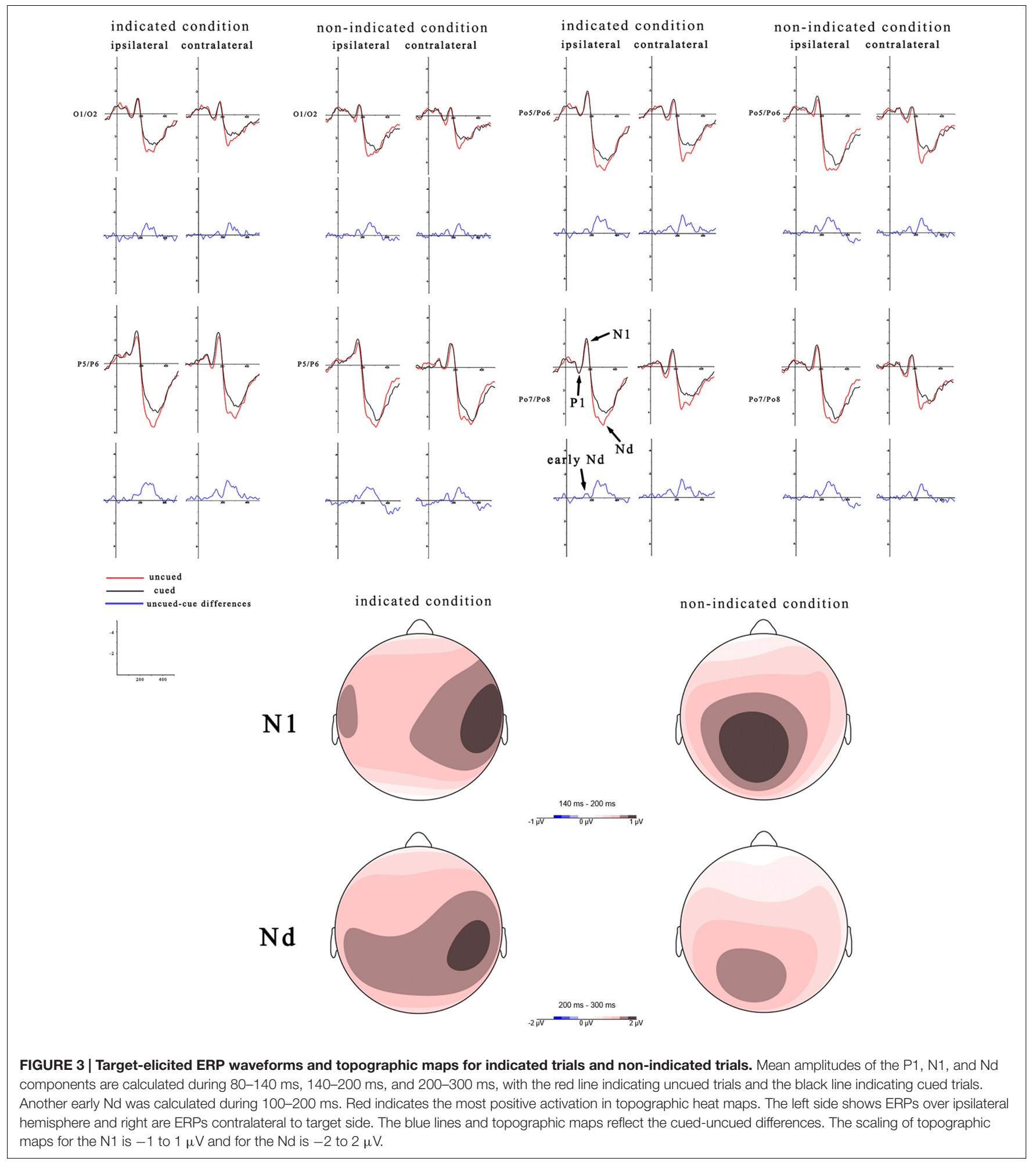

more negative on cued trials $(-0.44 \mu \mathrm{V})$ than uncued trials $(-0.22 \mu \mathrm{V})$ at both contralateral and ipsilateral sites. The main effect of central cueing was significant, $F(1,19)=5.2, p<0.05$, $\eta^{2}=0.22$, showing more negative amplitudes on indicated trials $(-0.46 \mu \mathrm{V})$ than non-indicated trials $(-0.21 \mu \mathrm{V})$. The main effect of laterality was not significant, $F(1,19)=0.41, p=0.53$, $\eta^{2}=0.02$. The interactions of peripheral cueing $\times$ central cueing, $F(1,19)=0.26, p=0.62, \eta^{2}=0.01$, of peripheral cueing $\times$ laterality, $F(1,19)=0.65, p=0.43, \eta^{2}=0.03$, of central cueing $\times$ laterality, $F(1,19)=0.49, p=0.5, \eta^{2}=0.03$, and of 
peripheral cueing $\times$ central cueing $\times$ laterality, $F(1,19)=0.83$, $p=0.37, \eta^{2}=0.04$, were not significant.

\section{DISCUSSION}

In order to further explore the relationship between automatic attentional orienting operated by a non-informative central cue and the IOR effect operated by a peripheral cue, we used a location task to simultaneously examine these two mechanisms from the aspects of behavior and electrophysiology. Consistent with our hypothesis, the present results replicated the findings of previous studies, showing that IOR was independent of involuntary spatial orienting in both behavioral performance and neural correlates. There were three main results: First, we found a significant main effect of IOR on the behavior and Nd components. Second, a significant central cueing effect was observed on the behavior and N1 component. Third, the peripheral cueing effect and the central cueing effect did not interact crucially with each other on the behavioral and electrophysiological levels. This indicated that IOR and involuntary orienting of spatial attention might not be mutually interrelated mechanisms.

In the present experiment, the peripheral cue succeeded in eliciting the IOR effect. For central cue, recent studies have two different explanations as mentioned in the introduction section. Green et al. (2013) found the facilitation of central cue only at the long duration cues for the short SOA, and they explained this with cue-target conflict. Nevertheless, Gayzur et al. (2014) found the facilitation of gaze cue at both the short and the long duration for both the short and the long SOA, and they employed an explanation of reflexive orienting. The paradigm of present experiment is different with Green et al. (2013) and Gayzur et al. (2014), but it is similar to Martín-Arévalo et al. (2013). Due to the different paradigms among Green et al. (2013), Martín-Arévalo et al. (2013), and Gayzur et al. (2014), the central cueing effects of Martín-Arévalo et al. (2013) and the present experiment were not pure arrow cue effect. Long SOA was used in the present experiment and Martín-Arévalo et al. (2013), the results showed resembled facilitation of the arrow cue. Green et al. (2013) who explained the result as cue-target conflict for non-predictive cues, and this cue-target conflict only appeared in the long duration (cue and target stimuli overlapped temporally). The present experiment did not make central cues and the target present simultaneously, thus the differences between indicated and non-indicated trials were not suitable for the explanation of cue-target conflict. Green et al. (2013) also did not find any cueing effect of arrow cue at the short duration, but Gayzur et al. (2014) found cueing effect at the short duration for the short SOA. This may lead to their different explanations for the central cueing effect. Moreover, for the long duration condition of reflexive orienting, MartínArévalo et al. (2013) found the central cueing effect at the long SOA and Gayzur et al. (2014) found that at both the short SOA and long SOA. It seemed that the spatial-conflict was supported only at the short SOA for the long duration. For the short duration condition, the central cueing effect was not affected by the return cue condition and this facilitation of non-predictive cues was significant up to $1440 \mathrm{~ms}$ (McKee et al., 2007). Tipples (2002) used short duration with an interval of 25 or $225 \mathrm{~ms}$ between non-predictive central cue and target, and found faster RTs which indicated automatic orienting at the valid location than those at the invalid location. The same pattern of data was observed when the present experiment adopted an interval of $300 \mathrm{~ms}$ after central cue. Thus, the non-predictive arrow used in this atypical paradigm may also reflect the automatic orienting. In addition, dissociation of attention from the attended location is considered as a core factory for generating the IOR effect (Posner and Cohen, 1984; Klein, 2000). Regardless of indicated or nonindicated positions, mean RTs or correct rate of the cued location was slower or lower than the uncued location which referred to an IOR effect. In other words, IOR has been observed when attention was not disengaged from the attended location. These results were highly compatible with previous studies (Berlucchi et al., 2000; Berger et al., 2005; Chica et al., 2006; Chica and Lupiáñez, 2009; Martín-Arévalo et al., 2013). Therefore, the present behavioral result disproved the "reorienting hypothesis" of IOR.

The underlying neural process of cueing effects has been widely investigated by ERPs (McDonald et al., 2009; Satel et al., 2012, 2014). Researchers were interested in exploring which components can better represent electrophysiological markers of IOR and facilitation in visual attention (Lupiáñez et al., 2006; Martín-Arévalo et al., 2016). However, there still remained many debates or contradictory results (Prime and Ward, 2006; Prime and Jolicoeur, 2009). In the present experiment, we first chose three ERP components (P1, N1, and Nd) to explore insights into the relationship of IOR and the central cueing effect. The P1 amplitudes did not have any effect. The N1 amplitudes were enhanced for both cued and indicated trials, but not for uncued and non-indicated trials. These two results were very similar to the findings of Satel et al. (2014). In their experiment, behavioral IOR was observed, but P1 reduction was absent in the central arrow condition. They explained that P1 reduction would disappear when the cue and target were not peripheral onsets. According to previous studies, this enhancement of N1 used to be associated with the facilitatory effect (Eimer, 1994; Doallo et al., 2005; Martín-Arévalo et al., 2014). However, considering the current 8 -ms difference between indicated and non-indicated trials during one SOA, this central cueing effect is not possible to unequivocally ascribe a small facilitatory effect to attention. Although the early N1 component seemed certainly plausible, it was not sure that this was the true $\mathrm{N} 1$ component because its time course was not close to the N1 peak. According to the difference waves, there seemed to be an early negative difference (Nd, subtracting invalidly cued amplitudes from validly cued amplitudes) component (McDonald et al., 1999) in the time range of 100-200ms, reflecting a single ERP component with a long duration. Thus, these effects of N1 component which appeared to start in the P1 interval needed to be further analyzed. The result of the early Nd was similar to McDonald et al. (1999) who investigated the same component (e.g., called posterior $\mathrm{Nd}$ in $120-200 \mathrm{~ms}$ ) and found this difference began at the onset of the $\mathrm{P} 1$ and continued for nearly $100 \mathrm{~ms}$ after the N1 peak. Certainly, 
this early posterior negative difference $(\mathrm{Nd})$ is unrelated to the N1. Two alternative interpretations will be discussed below. The early $\mathrm{Nd}$ effect spanning the P1 and N1 peaks (100-200 ms) was less positive on cued trials than that on uncued trials. This explanation was fitted well with perceptual inhibition which found smaller amplitudes of cued location for the early ERP components (Prime and Jolicoeur, 2009). On the other hand, this sustained $\mathrm{Nd}$ effect called reduced positivity that was also unrelated to the P1 amplitude. This different effect of peripheral cueing effect might indicate that it could arise from sensory refractoriness (McDonald et al., 1999).

Recently, Satel et al. (2014) found that Nd might be a more reliable neural marker of IOR. The Nd component reflecting a relation with IOR was often investigated at occipital electrodes during a time window ranging approximately from 200 to $300 \mathrm{~ms}$ (Prime and Ward, 2004, 2006; Wascher and Tipper, 2004; Satel et al., 2013). The Nd component had been related to the facilitatory effect for enhancement of the cued location (Eimer, 1993, 1994; Martín-Arévalo et al., 2014) and to the IOR effect for reduction of the cued location (Prime and Jolicoeur, 2009; Satel et al., 2012; Gutiérrez-Domínguez et al., 2014). The present experiment showed an inhibitory effect of $\mathrm{Nd}$. Compared to the uncued location, the amplitude of $\mathrm{Nd}$ was smaller at the cued location in both indicated and non-indicated trials. Moreover, interaction effect between peripheral cue and central cue was not significant. This performance of the $\mathrm{Nd}$ component indicated that IOR existed at the later process stage, which

\section{REFERENCES}

Berger, A., Henik, A., and Rafal, R. (2005). Competition between endogenous and exogenous orienting of visual attention. J. Exp. Psychol. Gen. 134, 207-221. doi: 10.1037/0096-3445.134.2.207

Berlucchi, G., Chelazzi, L., and Tassinari, G. (2000). Volitional covert orienting to a peripheral cue does not suppress cue-induced inhibition of return. J. Cogn. Neurosci. 12, 648-663. doi: 10.1162/089892900562408

Chica, A. B., Bartolomeo, P., and Lupiáñez, J. (2013). Two cognitive and neural systems for endogenous and exogenous spatial attention. Behav. Brain Res. 237, 107-123. doi: 10.1016/j.bbr.2012.09.027

Chica, A. B., Bartolomeo, P., and Valero-Cabré, A. (2011). Dorsal and ventral parietal contributions to spatial orienting in the human brain. J. Neurosci. 31, 8143-8149. doi: 10.1523/JNEUROSCI.5463-10.2010

Chica, A. B., and Lupiáñez, J. (2004). Inhibition of return without return attention. Psicothema 16, 248-254.

Chica, A. B., and Lupiáñez, J. (2009). Effects of endogenous and exogenous attention on visual processing: an inhibition of return study. Brain Res. 1278, 75-85. doi: 10.1016/j.brainres.2009.04.011

Chica, A. B., Lupiáñez, J., and Bartolomeo, P. (2006). Dissociating inhibition of return from endogenous orienting of spatial attention: evidence from detection and discrimination tasks. Cogn. Neuropsychol. 23, 1015-1034. doi: 10.1080/ 02643290600588277

Danziger, S., and Kingstone, A. (1999). Unmasking the inhibition of return phenomenon. Percept. Psychophys. 61, 1024-1037. doi: 10.3758/BF03207610

Doallo, S., Lorenzo-Lopez, L., Vizoso, C., Holguín, S. R., Amenedo, E., Bara, S., et al. (2004). The time course of the effects of central and peripheral cues on visual processing: an event-related potentials study. Clin. Neurophysiol. 115, 199-210. doi: 10.1016/S1388-2457(03)00317-1

Doallo, S., Lorenzo-Lopez, L., Vizoso, C., Holguín, S. R., Amenedo, E., Bará, S., et al. (2005). Modulations of the visual N1 component of event-related potentials by central and peripheral cueing. Clin. Neurophysiol. 116, 807-820. doi: 10.1016/j.clinph.2004.11.013 may refer to the decision-making process (McDonald et al., 1999).

In summary, the current experiment found a highly coincident result showing dissociation between IOR and automatic spatial orienting in the behavioral data and the potential neural marker of $\mathrm{Nd}$ for IOR. It was generally proposed that IOR could be observed without disengagement from the attended location.

\section{AUTHOR CONTRIBUTIONS}

FP and XW designed and coordinated the study; XW and LZ carried out experiments and data processes; XW drafted the manuscript and FP reviewed the manuscript. All authors gave final approval for publication.

\section{ACKNOWLEDGMENTS}

This work was supported by the Social Sciences Foundation of Jiangsu Province, China (14SHC006), the Humanities and Social Sciences Foundation of the Chinese Ministry of Education (12YJC190026), the Program for Twelfth FiveYear Plan of Educational Science of Jiangsu Province, China (C-a/2013/01/003), and the 2015 Annual Graduate Scientific Research Project of Regular Institutions of Higher Education of Jiangsu Province, China (KYZZ15_0355).

Eimer, M. (1993). Effects of attention and stimulus probability on ERPs in a Go/Nogo task. Biol. Psychol. 35, 123-138. doi: 10.1016/0301-0511(93)90009-W Eimer, M. (1994). An ERP study on visual spatial priming with peripheral onsets. Psychophysiology 31, 154-163. doi: 10.1111/j.1469-8986.1994.tb01035.x

Friesen, C. K., and Kingstone, A. (1998). The eyes have it! reflexive orienting is triggered by nonpredictive gaze. Psychon. Bull. Rev. 5, 490-495. doi: 10.3758/ BF03208827

Friesen, C. K., and Kingstone, A. (2003). Abrupt onsets and gaze direction cues trigger independent reflexive attentional effects. Cognition 87, B1-B10. doi: 10.1016/S0010-0277(02)00181-6

Funes, M. J., Lupiáñez, J., and Milliken, B. (2007). Separate mechanisms recruited by exogenous and endogenous spatial cues: evidence from a spatial Stroop paradigm. J. Exp. Psychol. Hum. Percept. Perform. 33, 348.

Gayzur, N. D., Langley, L. K., Kelland, C., Wyman, S. V., Saville, A. L., Ciernia, A. T., et al. (2014). Reflexive orienting in response to short- and long-duration gaze cues in young, young-old, and old-old adults. Atten. Percept. Psychophys. 76, 407-419. doi: 10.3758/s13414-013-0554-6

Green, J. J., Gamble, M. L., and Woldorff, M. G. (2013). Resolving conflicting views: gaze and arrow cues do not trigger rapid reflexive shifts of attention. Vis. Cogn. 21, 61-71. doi: 10.1080/13506285.2013.775209

Green, J. J., and Woldorff, M. G. (2012). Arrow-elicited cueing effects at short intervals: rapid attentional orienting or cue-target stimulus conflict? Cognition 122, 96-101. doi: 10.1016/j.cognition.2011.08.018

Gutiérrez-Domínguez, F. J., Pazo-Álvarez, P., Doallo, S., Fuentes, L. J., LorenzoLópez, L., and Amenedo, E. (2014). Vertical asymmetries and inhibition of return: effects of spatial and non-spatial cueing on behavior and visual ERPs. Int. J. Psychophysiol. 91, 121-131. doi: 10.1016/j.ijpsycho.2013.12.004

Hopfinger, J. B., and Mangun, G. R. (1998). Reflexive attention modulates processing of visual stimuli in human extrastriate cortex. Psychol. Sci. 9, 441-447. doi: 10.1111/1467-9280.00083

Ivanoff, J., and Taylor, T. L. (2006). Inhibition of return promotes stop-signal inhibition by delaying responses. Vis. Cogn. 13, 503-512. doi: 10.1080/ 13506280544000246 
Jones, A., and Forster, B. (2012). Reflexive attention in touch: an investigation of event related potentials and behavioural responses. Biol. Psychol. 89, 313-322. doi: 10.1016/j.biopsycho.2011.11.004

Jones, A., and Forster, B. (2014). Neural correlates of endogenous attention, exogenous attention and inhibition of return in touch. Eur. J. Neurosci. 40, 2389-2398. doi: 10.1111/ejn.12583

Jonides, J. (1981). "Voluntary versus automatic control over the mind's eye's movement," in Attention and Performance IX, ed. A. Baddeley (Hillsdale, NJ: Lawrence Erlbaum Associates), 187-203.

Klein, R. M. (2000). Inhibition of return. Trends Cogn. Sci. 4, 138-147. doi: 10.1016/ S1364-6613(00)01452-2

Luck, S. J. (2014). An Introduction to the Event-Related Potential Technique. Cambridge, MA: MIT press.

Lupiáñez, J. (2010). "Inhibition of return," in Attention and Time, eds K. Nobre and J. T. Coull (Cambridge, MA: Oxford University Press), 17-34.

Lupiáñez, J., Klein, R. M., and Bartolomeo, P. (2006). Inhibition of return: twenty years after. Cogn. Neuropsychol. 23, 1003-1014. doi: 10.1080/ 02643290600588095

MacInnes, W. J., and Klein, R. M. (2003). Inhibition of return biases orienting during the search of complex scenes. Sci. World J. 3, 75-86. doi: 10.1100/tsw. 2003.03

Martín-Arévalo, E., Chica, A. B., and Lupiáñez, J. (2014). Electrophysiological modulations of exogenous attention by intervening events. Brain Cogn. 85, 239-250. doi: 10.1016/j.bandc.2013.12.012

Martín-Arévalo, E., Chica, A. B., and Lupiáñez, J. (2016). No single electrophysiological marker for facilitation and inhibition of return: a review. Behav. Brain Res. 300, 1-10. doi: 10.1016/j.bbr.2015.11.030

Martín-Arévalo, E., Kingstone, A., and Lupiáñez, J. (2013). Is “inhibition of return” due to the inhibition of the return of attention? Q. J. Exp. Psychol. 66, 347-359. doi: 10.1080/17470218.2012.711844

McDonald, J. J., Hickey, C., Green, J. J., and Whitman, J. C. (2009). Inhibition of return in the covert deployment of attention: evidence from human electrophysiology. J. Cogn. Neurosci. 21, 725-733. doi: 10.1162/jocn.2009. 21042

McDonald, J. J., Ward, L. M., and Kiehl, K. A. (1999). An event-related brain potential study of inhibition of return. Percept. Psychophys. 61, 1411-1423. doi: 10.3758/BF03206190

McKee, D., Christie, J., and Klein, R. (2007). On the uniqueness of attentional capture by uninformative gaze cues: facilitation interacts with the simon effect and is rarely followed by ior. Can. J. Exp. Psychol. 61, 293-303. doi: 10.1037/ cjep2007029

Posner, M. I. (1980). Orienting of attention. Q. J. Exp. Psychol. 32, 3-25. doi: $10.1080 / 00335558008248231$

Posner, M. I., and Cohen, Y. (1984). “Components of visual orienting," in Attention and Performance X: Control of Language Processes, eds H. Bouma and D. Bouwhuis (Hillsdale, NJ: Erlbaum), 531-556.
Posner, M. I., Rafal, R. D., Choate, L. S., and Vaughan, J. (1985). Inhibition of return: neural basis and function. Cogn. Neuropsychol. 2, 211-228. doi: 10.1080/ 02643298508252866

Prime, D. J., and Jolicoeur, P. (2009). On the relationship between occipital cortex activity and inhibition of return. Psychophysiology 46, 1278-1287. doi: 10.1111/ j.1469-8986.2009.00858.x

Prime, D. J., and Ward, L. M. (2004). Inhibition of return from stimulus to response. Psychol. Sci. 15, 272-276. doi: 10.1111/j.0956-7976.2004.00665.x

Prime, D. J., and Ward, L. M. (2006). Cortical expressions of inhibition of return. Brain Res. 1072, 161-174. doi: 10.1016/j.brainres.2005.11.081

Ruz, M., and Castillo, J. L. (2002). A review of attentional capture: on its automaticity and sensitivity to endogenous control. Psicológica 23, 283-310.

Satel, J., Hilchey, M. D., Wang, Z., Reiss, C. S., and Klein, R. M. (2014). In search of a reliable electrophysiological marker of oculomotor inhibition of return. Psychophysiology 51, 1037-1045. doi: 10.1111/psyp.12245

Satel, J., Hilchey, M. D., Wang, Z., Story, R., and Klein, R. M. (2013). The effects of ignored versus foveated cues upon inhibition of return: an event-related potential study. Atten. Percept. Psychophys. 75, 29-40. doi: 10.3758/s13414-0120381-1

Satel, J., Wang, Z., Hilchey, M. D., and Klein, R. M. (2012). Examining the dissociation of retinotopic and spatiotopic inhibition of return with eventrelated potentials. Neurosci. Lett. 524, 40-44. doi: 10.1016/j.neulet.2012.07. 003

Taylor, T. L., and Klein, R. M. (2000). Visual and motor effects in inhibition of return. J. Exp. Psychol. Hum. Percept. Perform. 26, 1639-1656.

Tipples, J. (2002). Eye gaze is not unique: automatic orienting in response to uninformative arrows. Psychon. Bull. Rev. 9, 314-318. doi: 10.1016/j.beproc. 2009.03.018

Wang, Z., Satel, J., and Klein, R. M. (2012). Sensory and motor mechanisms of oculomotor inhibition of return. Exp. Brain Res. 218, 441-453. doi: 10.1007/ s00221-012-3033-8

Wascher, E., and Tipper, S. P. (2004). Revealing effects of noninformative spatial cues: an EEG study of inhibition of return. Psychophysiology 41, 716-728. doi: 10.1111/j.1469-8986.2004.00198.x

Conflict of Interest Statement: The authors declare that the research was conducted in the absence of any commercial or financial relationships that could be construed as a potential conflict of interest.

Copyright (c) 2017 Pan, Wu and Zhang. This is an open-access article distributed under the terms of the Creative Commons Attribution License (CC BY). The use, distribution or reproduction in other forums is permitted, provided the original author(s) or licensor are credited and that the original publication in this journal is cited, in accordance with accepted academic practice. No use, distribution or reproduction is permitted which does not comply with these terms. 